\section{¿Mitomicina C o 5-FU en la manipulación con aguja de ampollas fracasadas? \\ Mitomycin $\mathrm{C}$ versus 5-FU in failing blebs needling}

Estimado Sr. Director:

Quisiera expresar desde estas líneas mi felicitación al equipo que dirige la Dra. Esperanza Gutiérrez por los resultados obtenidos en la serie que han publicado recientemente en su artículo titulado «Repermeabilización mediante revisión con aguja de ampollas de filtración con fracaso tardío tras cirugía de glaucoma» (1).

Aunque los resultados son fruto de un estudio retrospectivo con las limitaciones que esto puede suponer, obtienen una importante tasa de éxitos en ampollas previamente fracasadas, ya que la media de tiempo transcurrido desde la cirugía filtrante hasta su revisión con 5-FU es de 67,3 meses, incluyendo un caso de fracaso 23 años tras la cirugía.

En este trabajo reseñan, desde el punto de vista de estadística descriptiva, el tiempo transcurrido desde el acto quirúrgico hasta el fracaso de ampolla pero, parece que no tiene relevancia significativa. En otros trabajos si este intervalo es más corto, se considera como uno de los factores que más contribuye al éxito de la repermeabilización, pudiendo incluso ayudar a la selección previa de los pacientes.

A nivel personal, dejé de utilizar el 5-FU hace muchos años por las complicaciones epiteliales, las molestias subjetivas que ocasionan al paciente y la necesidad de retratamientos $(2,3)$. En nuestra experiencia personal ha sido más rentable con la Mitomicina $\mathrm{C}$ asociada a manipulación con aguja en casos donde no se había empleado intraoperatoriamente, lo que reduce el riesgo de ampollas cadavéricas (4).

Sin embargo, en el presente artículo las complicaciones son menos frecuentes y menos severas que las de otras series publicadas, lo que hace, teniendo en cuenta la seriedad que caracteriza a este grupo de trabajo de Glaucoma del Hospital 12 de Octubre, que otros oftalmólogos, entre los que me incluyo nos volvamos a plantear realizar las revisiones de ampollas con 5-FU en fracasos tardíos (1).

Finalmente reitero mi agradecimiento a este grupo, por seguir trabajando en esta línea que tanto nos aporta a los que diariamente sufrimos los avatares de la cirugía de glaucoma y sus muchas veces impredecibles y difíciles post-operatorios, no sin antes considerar que, todos necesitamos más estudios prospectivos y de doble brazo con ambos antifibroblásticos, para valorar resultados a largo plazo.

Cabarga del Nozal C Doctora en Medicina. Hospital Ramón y Cajal. Madrid. E-mail: ccabarga.hrc@ salud.madrid.org

\section{BIBLIOGRAFÍA}

1. Perucho-Martínez S, Gutierrez-Díaz E, Montero-Rodríguez M, Mencia-Gutierrez E, Lago-Llinas MD. Repermeabilización mediante revisión con aguja de ampollas de filtración con fracaso tardio tras cirugía de glaucoma. Arch Soc Esp Oftalmol 2006; 81: 517-522.

2. Broadway DC, Bloom PA, Bunce C, Thiagarajan M, Khaw PT. Needle revision of failing and failed trabeculectomy blebs with adjunctive 5-fluorouracil: survival analysis. Ophthalmology 2004; 111: 665-673.

3. Greenfield DS, Millar MP, Suner IJ, Palmberg PS. Needle elevation of the scleral flap for failing filtration blebs after trabeculectomy with mitomycin C. Am J Ophthalmol 1996; 122: 195-204.

4. Gutierrez-Ortiz C, Cabarga C, Teus MA. Prospective evaluation of preoperative factors associated with successful mitomycin C needling of failed filtration blebs. J Glaucoma 2006; 15: 98-102.

Réplica

Estimado Sr. Director:

Respecto a la carta remitida por la Dra. Carmen Cabarga del Nozal, que agradecemos sinceramente, quisiéramos comentar algunos puntos.

Nuestro trabajo estudia las posibilidades de recuperar la filtración en ampollas inicialmente funcionantes y que fracasan de forma tardía, y todos los casos que presentamos son ojos con cirugía filtrante realizada más de 6 meses antes (1). Por lo tanto, es una situación totalmente distinta a la de otros trabajos (2), que incluyen pacientes recién operados. Estoy de acuerdo en que una de las claves del éxito para conseguir una buena ampolla de filtración es conseguir y mantener el paso de acuoso a través de la misma, antes de que se establezcan adherencias conjuntivales. En este sentido, es fundamental que la revisión con aguja, asociada al antimetabolito de elección, se haga de forma precoz, aunque, en nues- 
tra experiencia, si existe gran congestión e inflamación conjuntivales, la manipulación con aguja puede ser incluso contraproducente, ya que exacerba esta situación y favorece la fibrosis. Por ello, en esos casos, preferimos controlar la PIO con tratamiento médico y demorar la revisión hasta que cede la inflamación. Creemos que la selección de los pacientes, basada en las características de la ampolla, es el factor fundamental para el éxito de este procedimiento, y que puede ser eficaz incluso largo tiempo tras la cirugía, que es lo que pretendíamos demostrar en nuestro estudio.

En cuanto a la elección del antimetabolito, no cabe duda de que el uso de 5-fluorouracilo (5-FU) es más laborioso, ya que requiere más aplicaciones, y por tanto, más revisiones. No obstante, lo preferimos en base a nuestra experiencia, ya que es el antimetabolito que usamos de forma intraoperatoria. Nuestro interés es el mantenimiento de la visión a largo plazo, y estamos convencidos de que, aunque la mitomicina (MMC) consigue menores presiones intraoculares (PIO), sus complicaciones también son mayores, lo cual compensa la supuesta inferioridad del 5-FU, como queda demostrado en los artículos del grupo del Moorfields Eye Hospital $(3,4)$. Por otra parte, la toxicidad epitelial puede minimizarse tomando ciertas precauciones, como son evitar el reflujo al inyectar, lavar la superficie ocular tras la inyección y asociar el uso frecuente de colirios humectantes.

Sin embargo, reconocemos que la MMC está demostrando buenos resultados y escasas complicaciones en su aplicación subconjuntival asociada a revisión con aguja, como demuestra la propia Dra.
Cabarga (5), y estamos de acuerdo en que un estudio prospectivo comparando ambos antimetabolitos sería una idea excelente, ya que nos proporcionaría la evidencia necesaria para una elección idónea.

Gutiérrez Díaz E, Montero Rodríguez M, Mencía Gutiérrez E Licenciados en Medicina Servicio de Oftalmología

Hospital Universitario 12 de Octubre de Madrid E-mail: egutierrez.hdoc@salud.madrid.org

\section{BIBLIOGRAFÍA}

1. Perucho-Martínez S, Gutiérrez-Díaz, E, Montero-Rodríguez, M, Mencía-Gutiérrez E, Lago-Llinás MD. Repermeabilización mediante revisión con aguja de ampollas de filtración con fracaso tardio tras cirugía de glaucoma. Arch Soc Esp Oftalmol 2006; 81: 517-522.

2. Li G, Kasner O. Review of consecutive phacotrabeculectomies supplemented with early needle revision and antimetabolites. Can J Ophthalmol 2006; 41: 457-463.

3. Membrey WL, Poinoosawmy DP, Bunce C, Hitchings RA. Glaucoma surgery with or without adjunctive antiproliferatives in normal tension glaucoma: 1 intraocular pressure control and complications. Br J Ophthalmol 2000; 84: 586-590.

4. Membrey WL, Bunce C, Poinoosawmy DP, Fitzke FW, Hitchings RA. Glaucoma surgery with or without adjunctive antiproliferatives in normal tension glaucoma: 2 Visual field progression. Br J Ophthalmol 2001; 85: 696701.

5. Gutiérrez-Ortiz C, Cabarga C, Teus MA. Prospective evaluation of preoperative factors associated with successful mitomycin $C$ needling of failed filtration blebs. J Glaucoma 2006; 15: 98-102. 